\title{
Coming soon: an expansion of the Journal's critical care offerings
}

\author{
Hilary P. Grocott, MD, FRCPC, FASE
}

Received: 7 July 2017/Accepted: 13 July 2017/Published online: 21 July 2017

(c) Canadian Anesthesiologists' Society 2017

In the spring of this year, the Canadian Anesthesiologists' Society (CAS) and the Canadian Critical Care Society (CCCS) signed an agreement making the Canadian Journal of Anesthesia the official journal of the CCCS. Critical care has always been a fundamental part of the Journal's mission of "Excellence in research and knowledge translation in anesthesia, pain, perioperative medicine, and critical care", and we look forward to expanding our presence in this area. Readers will find this new partnership reflected on the Journal's front cover which now features the new bilingual logo of the CCCS. The Journal's Editorial Board membership has similarly evolved with the addition of an Associate Editor (Dr. Sangeeta Mehta from the University of Toronto) and another Editorial Board Member (Dr. Donald Griesdale from the University of British Columbia) who will focus on critical care submissions.

As always, the Journal continues to welcome content in all fields of anesthesiology, pain, and perioperative medicine, and now further supports those areas of interest with an expanded focus on critical care. Indeed, some of this material has recently appeared in the Journal, predating this more formal change, for example, the recently published WikiRecs guidelines on vasopressor use and hemodynamic goals in septic shock put forth, in part, by the CCCS. ${ }^{1-3}$

Partnership with the CCCS is anticipated to offer expanded content to readers, solidify the relationship between the specialties of anesthesiology and critical

H. P. Grocott, MD, FRCPC, FASE ( $\square)$

Department of Anesthesia, St. Boniface Hospital, University of Manitoba, CR3008 - 369 Tache Avenue, Winnipeg,

MB R2H 2A6, Canada

e-mail: hgrocott@sbgh.mb.ca care, and strengthen the overall impact of the Journal. Anesthesiologists and critical care specialists share common interests in a number of areas, including airway management, ventilation, resuscitation, and sedation, to name but a few. Sharing our knowledge in the Journal can only benefit the management and outcomes of the patients in our care.

\section{Prochainement : l'expansion du contenu en soins intensifs du Journal}

La Société canadienne des anesthésiologistes (SCA) et la Société canadienne de soins intensifs (SCSI) ont signé un accord ce printemps selon lequel le Journal canadien d'anesthésie devient la revue officielle de la SCSI. Les soins intensifs ont toujours fait partie intégrante de la mission du Journal, comme l'indique son énoncé : "L'excellence en recherche et en transfert des connaissances liées à l'exercice clinique de l'anesthésie, $d u$ contrôle de la douleur, de la médecine périopératoire et des soins intensifs ». Nous nous réjouissons donc de notre développement dans ce domaine. Pour le lecteur, ce nouveau partenariat sera visible sur la couverture du Journal : désormais le nouveau logo bilingue de la SCSI y figurera. Le Comité de rédaction du Journal a lui aussi été modifié pour accueillir deux nouveaux membres, soit la Dre Sangeeta Mehta, de l'Université de Toronto, en qualité de rédactrice adjointe, et le Dr Donald Griesdale, de l'Université de Colombie-Britannique, en tant que membre du Comité de rédaction; ces deux nouveaux membres se 
concentreront sur les soumissions portant sur les soins intensifs.

Comme toujours, le Journal continuera d'accepter du contenu touchant tous les domaines pertinents à l'anesthésiologie, à la douleur et à la médecine périopératoire, mais avec une emphase plus concrète sur les soins intensifs. En fait, certains de ces thèmes ont déjà été récemment abordés dans le Journal avant même ce changement formel, notamment dans les directives WikiRecs récemment publiées concernant l'utilisation des vasopresseurs et les cibles hémodynamiques présentées, en partie, par la SCSI. ${ }^{1-3}$

Grâce à ce partenariat avec la SCSI, nous espérons élargir l'offre de contenu pour le lecteur, approfondir la relation entre les spécialités de l'anesthésiologie et des soins intensifs, et augmenter l'impact global du Journal. Les anesthésiologistes et les intensivistes partagent des intérêts communs dans plusieurs domaines, notamment la prise en charge des voies aériennes, la ventilation, la réanimation, ou encore la sédation, pour ne nommer que ceux-ci. Le partage de nos connaissances dans le Journal ne peut qu'être avantageux pour la prise en charge et les devenirs des patients dont nous nous occupons.
Conflicts of interest None declared.

Editorial responsibility This submission was handled by Dr. Gregory L. Bryson, Deputy Editor-in-Chief, Canadian Journal of Anesthesia.

Conflit d'intérêt Aucun.

Responsabilité éditoriale Cet article a été traité par Dr Gregory L. Bryson, rédacteur en chef adjoint, Journal canadien d'anesthésie.

\section{References}

1. Rochwerg B, Hylands M, Moller M, et al. CCCS-SSAI WikiRecs Clinical Practice Guideline: vasopressor blood pressure targets in critically ill adults with hypotension. Can J Anesth 2017; 64: 763-5.

2. Rochwerg B, Hylands M, Moller M, et al. CCCS-SSAI WikiRecs Clinical Practice Guideline: vasopressors in early traumatic shock. Can J Anesth 2017; 64: 766-8.

3. Siemieniuk RA, Guyatt GH. The next frontier in critical care guidelines: rapid and trustworthy recommendations. Can J Anesth 2017; 64: 689-92. 